\title{
CANCER AS A POTENTIAL CAUSE OF CRYPTOGENIC STROKE: A CASE REPORT
}

\author{
Mirjana Jovićević1,2, Sonja Rajić1, Željko Živanović1,2, Tamara Rabi-Žikić1,2, Ivana \\ Divjak $^{1,2}$. Marija Žarkov ${ }^{1,2}$, Svetlana Ruzička-Kaloci ${ }^{1,2}$, Aleksandar Jovanović1,2,Marija \\ Milićević ${ }^{1}$, Goran Knezović ${ }^{1}$
}

1.Clinical of Neurology, Clinical Center od Vojvodina, Novi Sad, Serbia
2.Faculty of Medicine, University of Novi Sad, Serbia

Background: Ischemic strokes of undetermined etiology can be non-embolic and embolic, which include paroxysmal atrial fibrillation, minor cardiac causes, atheroembolism, paradoxical embolism through patent foramen ovale (PFO) and cancer associated stroke.Hypercoagulability accounts as the most important mechanism of malignancy caused stroke.Once the traditional risk factors are excluded as the cause of stroke, we can refer to d-dimer levels as the surrogate marker for hypercoagulability.

Case Report: A 69-year old woman was admitted to Neurology Clinic for sudden onset of right-sided weakness and difficulties of speech. She had a history of hypertension and diabetes. Physical examination confirmed neurological deficit. Duplex ultrasonography scan of carotid arteries was normal. Computed tomography (CT) of the brain showed infarction of the right cerebellum as well as frontoparietal infarction on the left side. Since vaginal bleeding and ascites were observed, the patient underwent CT of the abdomen that revealed tumor mass located in the pelvis. Tumor markers for ovarian cancer (CA 125, HE4) were elevated. During hospitalization she developed signs for deep vein thrombosis that was confirmed by ultrasound examination. The patient was treated with low molecular weight heparin and refered to gynecologist for further investigation.

Conclusion: LMWH has been widely used for treating stroke patients with cancer, but its efficacy has not been well studied. Direct oral anticoagulants (DOAC) are being considered for treatment of malignancy related cryptogenic stroke. The use of DOAC can certainly lead to better compliance but further studies are needed to determine its safety and efficacy.

1. Fonseca AC, Ferro JM. Cryptogenic stroke. European Journal of Neurology 2015, 22: 618-23.

2. Schwarzbach CJ et al. Stroke and Cancer: The Importance of Cancer-Associated Hypercoagulation as a Possible Stroke Etiology. Stroke. 2012;43:302934.

3. Nam KW et al. Treatment of Cryptogenic Stroke with Active Cancer with a New Oral Anticoagulant. J Stroke Cerebrovasc Dis. 2017;26(12):2976-80.

Mirjana Jovicevic, MD, PhD Clinic of Neurology, Clinical center of Vojvodina, Hajduk Veljkova 1-7 $+381648059293$ 